\title{
Cambio ambiental y chamanización de las nuevas prácticas religiosas entre los yánesha (piedemonte peruano)
}

Changement environnemental et chamanisation des nouvelles pratiques

religieuses chez les Yánesha (piémont péruvien)

Environmental change and chamanization of new religious practices among

Yánesha people (Peruvian eastern slopes)

\section{Céline Valadeau}

\section{(2) OpenEdition}

Journals

Edición electrónica

URL: http://journals.openedition.org/bifea/10258

DOI: 10.4000/bifea.10258

ISSN: 2076-5827

\section{Editor}

Institut Français d'Études Andines

\section{Edición impresa}

Fecha de publicación: 8 diciembre 2018

Paginación: 313-333

ISSN: 0303-7495

Referencia electrónica

Céline Valadeau, «Cambio ambiental y chamanización de las nuevas prácticas religiosas entre los yánesha (piedemonte peruano) », Bulletin de l'Institut français d'études andines [En línea], 47 (3) | 2018, Publicado el 08 diciembre 2018, consultado el 04 noviembre 2020. URL : http:// journals.openedition.org/bifea/10258; DOI : https://doi.org/10.4000/bifea.10258

Les contenus du Bulletin de l'Institut français d'études andines sont mis à disposition selon les termes de la licence Creative Commons Attribution - Pas d'Utilisation Commerciale - Pas de Modification 4.0 International. 


\title{
Cambio ambiental y chamanización de las nuevas prácticas religiosas entre los yánesha (piedemonte peruano)
}

\author{
Céline Valadeau ${ }^{*}$
}

\begin{abstract}
Resumen
A través del análisis de los discursos sobre el cambio ambiental, este artículo permite reflexionar acerca de la reorganización de las prácticas religiosas entre los yánesha. La discusión pone en perspectiva unas referencias históricas sobre la estructura político-religiosa de este grupo étnico durante el siglo pasado e informaciones etnográficas recientes provenientes de dos comunidades: una cristiana evangélica y otra adventista. Así, queremos identificar los potenciales mecanismos de incorporación de las alocuciones evangélicas relacionadas con una «conversión ecológica», para entender mejor la variabilidad de algunas formas narrativas identificadas que abordan las relaciones entre los seres vivos. Por último, y a la luz de estas palabras, deseamos examinar un eventual proceso de chamanización de esas nuevas prácticas religiosas entre los yánesha.
\end{abstract}

Palabras clave: evangelismo, cambio ambiental, relaciones interespecíficas, yánesha, piedemonte peruano

\section{Changement environnemental et chamanisation des nouvelles pratiques religieuses chez les Yánesha (piémont péruvien)}

\section{Résumé}

L'analyse des discours sur la notion de changement environnemental permet d'apporter une réflexion sur l'idée d'un éventuel remaniement des pratiques religieuses chez les Yánesha. La discussion met en perspective certaines références historiques traitant de la structure politico-religieuse de ce groupe au cours du siècle dernier, et des informations ethnographiques récentes recueillies dans deux communautés : I'une évangélique et l'autre adventiste. Ainsi, nous cherchons à identifier l'existence

Investigadora asociada al IFEA, UMIFRE 17 MAEDI/CNRS USR 3337 América Latina. E-mail: celine. valadeau@protonmail.fr 
de potentiels mécanismes d'incorporation des prédications évangéliques traitant de la « conversion écologique » dans le but de comprendre la variabilité de quelques formes narratives mentionnant certaines des relations que les vivants possèdent entre eux. A la lumière de ces mots, I'hypothèse d'un éventuel processus de chamanisation des nouvelles pratiques religieuses adoptées par les Yánesha est examinée.

Mots-clés : Évangélisme, changement environnemental, relations interspécifiques, Yánesha, piémont péruvien

\title{
Environmental change and chamanization of new religious practices among Yánesha people (Peruvian eastern slopes)
}

\begin{abstract}
The analysis of discourses by paying a special attention to the notion of environmental change makes it possible to consider a potential reorganization of religious practices among the Yánesha. The discussion puts into perspective some historical references dealing with the political-religious structure of this group in the last past century, and recent ethnographic information collected in two communities: one Evangelical and another Adventist. Thus, the existence of potential mechanisms of incorporation of evangelical preaching dealing with an "ecological conversion" will be considered in order to understand the variability of several narrative forms mentioning various of the relationships types that the living have between them. Given this background, the hypothesis of a possible process of shamanization of the new religious practices adopted by the Yánesha is investigated. Possible process of chamanization of the new religious practices adopted by the Yánesha is investigated.
\end{abstract}

Keywords: Evangelism, environmental change, interspecies relationships, Yánesha, Peruvian eastern slopes

Desde los primeros contactos entre pueblos indígenas y misioneros, algunos predicadores elegían a los aspirantes más entusiastas para predicarles y bautizarlos. A partir de ese entonces se inició la incorporación de las ideas religiosas misioneras a las comunidades indígenas. Los acercamientos fueron establecidos, en primer lugar, por unos encargados de enseñar la doctrina católica. En la actualidad, se constata la propagación de algunos grupos de distintas inspiraciones religiosas, en su mayoría evangélicos y herederos de la Reforma protestante del siglo XVI. En la Selva central del Perú, la presencia de las Iglesias evangélicas y adventistas está en aumento desde hace aproximadamente un siglo. El impacto de su presencia, sus acciones y discursos entre los pueblos indígenas de esa región son notorios y han dejado huella en el paisaje político-religioso de dichos pueblos, a tal punto que podemos hablar de nuevas prácticas religiosas indígenas. Recientemente, varias instancias religiosas se han interesado por la problemática del cambio climático y sus repercusiones; por esta razón se vuelven a examinar las nociones que tratan de la relación que los humanos mantienen con la naturaleza (o especies vivas) en la cual se inscriben y con la cual interactúan. Ahora, si nos enfocamos en las 
formas de concebir la coexistencia humana con su entorno, entre el pensamiento difundido por las Iglesias y el de los indígenas, las divergencias son fundamentales y radicales. La concepción del medioambiente es muy distinta, incluso opuesta. Sin embargo, ciertos discursos indígenas llaman la atención por llevar elementos discursivos que provienen de ambos lados.

A lo largo de este artículo examinaremos los discursos, tanto evangélicos como indígenas, que tratan sobre el cambio ambiental, con el fin de abordar una reflexión acerca de las dinámicas de las prácticas religiosas entre los yánesha. La discusión articulará unas referencias históricas sobre su estructura político-religiosa durante el siglo pasado e informaciones etnográficas recientes provenientes de dos comunidades: una cristiana evangélica, Azulis, y otra adventista, Loma Linda-Puerto Laguna Raya (fig. 1). Nuestra hipótesis de trabajo está basada en la existencia tanto de dinámicas de incorporación como de alejamiento de las ideas evangélicas relacionadas con la representación de una «conversión ecológica» dentro del discurso indígena. Así, nos proponemos identificar formas narrativas y relatos que tratan de las especies vivas y que son predicados, por un lado, por los misioneros evangélicos y adventistas y, por otro lado, por los evangélicos y adventistas yánesha. Por último, queremos analizar cuál podría ser un eventual proceso de chamanización de esas nuevas prácticas religiosas entre los yánesha.

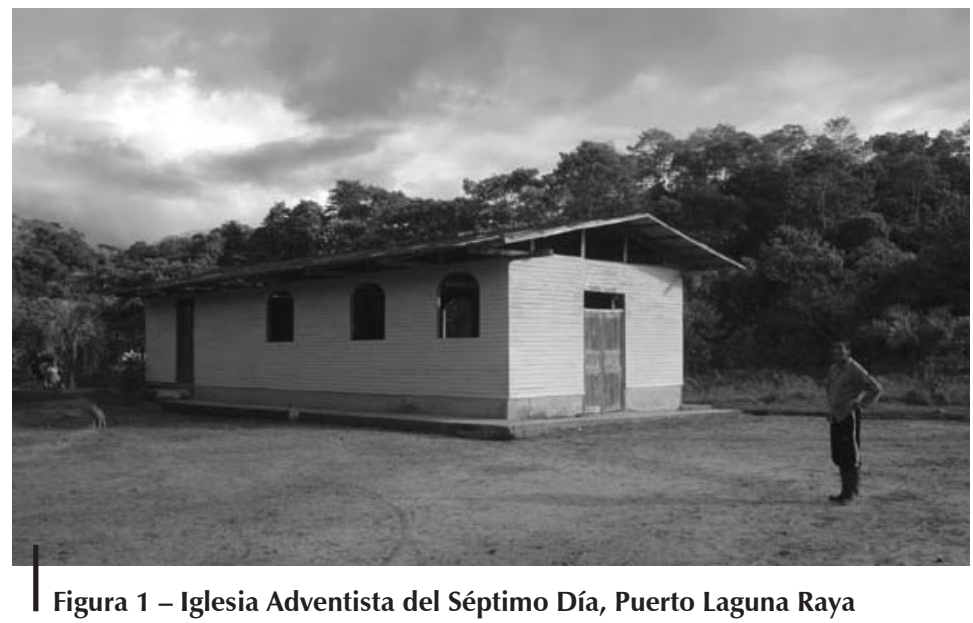

(C) Céline Valadeau, 2008

\section{ALGUNOS PUNTOS SOBRE LA HISTORIA DEL LIDERAZGO YÁNESHA}

Los yánesha (anteriormente Ilamados «amuesha») tienen filiación con la familia lingüística arawak. Según el censo de 2007, alrededor de 7550 personas yánesha viven en el piedemonte de la Selva central peruana ubicado en las provincias de Oxapampa (Pasco), Chanchamayo (Junín) y Puerto Inca (Huánuco) (según el censo 
de 1993 eran 8980). Las comunidades están ubicadas a una altitud que varía entre 200 y 1800 metros sobre el nivel del mar en las márgenes de los ríos Pichis, Palcazú, Pachitea, Huancabamba, Cacazú, Chorobamba y Yurinaqui. El territorio actual ha sido modificado respecto al ancestral. A inicios del siglo XVII, es decir, antes de la colonización por parte de misionarios y militares del piedemonte de la Selva central peruana, los yánesha residían en un corredor de valles intermontanos. Allí, se ubicaban en asentamientos dispersos que fueron constituidos por varias unidades domésticas emparentadas entre sí. Cada asentamiento era autónomo económica y políticamente. Estaban vinculados a través del intercambio de bienes como la sal de piedra, las alianzas matrimoniales y algunas celebraciones rituales (Benavides \& Pariona, 2002: 353). La pérdida de su territorio en beneficio de los colonos fue el resultado de varias incursiones: penetraciones militares (1847) e instalación de colonias austro-alemanas (finales del siglo XIX). Entre 1847 y 1900, la mayor parte de la población yánesha se desplazó hacia tierras más bajas (Santos-Granero, 1998: 135).

Según la documentación misionera, la organización político-religiosa yánesha en la época de Juan Santos (1710-1756) contaba con «sacerdotes» (San Joseph, 1716 citado en Santos-Granero, 2004: 196; Smith, 1999: 70). El sacerdote era considerado como una persona dedicada a los actos de culto y en ocasiones como un intermediario entre los miembros de una comunidad y la divinidad adorada. La literatura contemporánea relata la existencia de un tipo de sacerdotes yánesha llamados kornesha', que tenían funciones políticas, además de las religiosas concedidas por los españoles, sobre un conjunto de familias extensas pertenecientes a diferentes parcialidades. Tenían a su cargo centros ceremoniales que comprendían un templo (puerahua, lit. casa sagrada). Recientemente, a fines del siglo XIX, los exploradores mencionaron templos asociados a ferrerías ubicados en los intersticios del espacio social yánesha (Smith, comunicación personal, 2018). Los centros ceremoniales eran también lugares de peregrinaje que atraían no solo a la población yánesha, sino también a miembros de otras etnias como los asháninka (Perené y Pichis), ashéninka (Gran Pajonal) y konibo (Pachitea y Alto Ucayali) (Smith, 1977: 256). Su posición de mediadores entre los humanos y las divinidades hacía de los kornesha' un tipo de profetas, lo que explica su amplia influencia moral y política que iba más allá de las fronteras de la propia etnia. Los sacerdotes descritos por los misioneros eran de cierta manera los intermediarios entre la gente y los ancestros, entre ellos la divinidad Sol Yompor Ror (Smith, 1977: 101). Sus conocimientos, sabidurías y poderes eran considerados grandes; en coyunturas de conflicto, los kornesha' eran consultados por los guerreros (amcha'tareth, lit. al que se le mira con respeto). Según Santos-Granero, este particular tipo de organización sociopolítica constituye un desarrollo poscolonial. Tanto la influencia misionera como la de Juan Santos Atahualpa, a partir de 1709 y 1742 respectivamente, provocaron que el proceso de diferenciación entre chamanes y sacerdotes fuera ahondándose y culminara en la cristalización de la figura del kornesha' (figura político-religiosa), quien se distingue desde entonces del chamán (figura curandero-hechicera) y del líder guerrero (figura secular) (Santos-Granero, 2004: 208). 
Es posible que por aquel entonces tanto sacerdotes como chamanes pasasen por un mismo entrenamiento que luego se diferenció por influencias misioneras, junto con otros asuntos (Santos-Granero, 2004: 208). Sabiendo que desde entonces los roles de cada uno eran muy diferentes, unas similitudes pueden ser subrayadas, tomando en cuenta que las fuentes de sus poderes también eran distintas. Al igual que los chamanes (pa'llerr), los kornesha' usaban la coca y el tabaco y practicaban vigilias rituales (che'chen, lit. mantenerse despierto durante horas en la oscuridad). Ambos recibían un entrenamiento específico durante el cual ciertos espíritus les «enseñaban» sus cantos. Se dice que una persona que busca acceso a la música de las divinidades está «en un estado de anticipación de una revelación divina» (a'muerorrterra) (Smith, 1977: 153). Poco a poco, los kornesha' aprendían a controlar el poder del tabaco y practicaban una rutina rigurosa de vigilias y otras fórmulas rituales. A medida que acumulaban el poder divino, aumentaban sus conocimientos e influencias políticas (Smith, 1977: 244). Por otro lado, los aprendices chamanes entraban en reclusión y practicaban rituales (toreteñets) acumulando el poder de curar o hechizar. Las revelaciones auditivas debían ser memorizadas con exactitud (Smith, 1977: 180). Si lograban memorizar dichas revelaciones, los chamanes adquirían los espíritus primordiales de ciertas especies animales y/o vegetales y los kornesha' los cantos sagrados de los espíritus menores Mellañotheñ (yomporma'thtateñets, lit. esperar la manifestación de un dios). Los kornesha' transmitían después estos cantos a sus seguidores como mensajes de alta importancia, siempre introducidos por las palabras:

Escuchen a Yompor Ror y tengan buenos pensamientos. No tengan pensamientos malos. Deben vivir correctamente para que así les vaya bien. Además, los kornesha' garantizaban el éxito de las actividades de producción de recursos y la reproducción de la gente que vivía a su lado, en los centros ceremoniales. Con la muerte del último kornesha', alrededor del año 1974, Mekhllatarren, Miguel Grande, los templos fueron abandonados.

Entre 1969 y 1970 apareció un nuevo tipo de organización sociopolítica con ciertas influencias occidentales. Las reivindicaciones indígenas encontraron un respaldo en la Ley de Comunidades Nativas y de Desarrollo Agrario de la Selva y de Ceja de Selva de 1974, aunque correspondía también a un control del Estado sobre el territorio amazónico. En esta época, cada comunidad yánesha tuvo que organizarse a través de una junta directiva compuesta por un jefe o delegado (amcharets), un secretario y un tesorero (art. 22). Aunque las formas de autoridad reales no son exactamente el reflejo de esa reciente organización, sigue siendo importante asegurar una interacción con las instituciones estatales. Hoy, la terminología kornesha' es utilizada para denominar al representante de la organización política FECONAYA (Federación de Comunidades Nativas Yaneshas) sin ninguna dimensión religiosa perceptible. Los llamaremos kornesha' contemporáneos para diferenciarlos de los antiguos kornesha'. 


\section{RED DE SERVICIOS MISIONEROS EN LA ALTA AMAZONÍA}

Antes del contacto con las incursiones religiosas contemporáneas, los yánesha tuvieron acercamientos con los franciscanos y dominicos¹ (Santos, 1986: 121). A finales del siglo XVI, se ordena al Virrey no utilizar más los pictogramas, sino enseñar a «esa gente [...] nuestra lengua castellana» para entender mejor el santo evangelio (Quintana, 2004: 44). Desde finales del siglo XVII, los franciscanos fueron conscientes del fracaso de las expediciones religiosas (Amich, 1975: 79 y 94). Posteriormente, en las últimas décadas del siglo XIX, estas expediciones religiosas fueron estigmatizadas porque significaban una ocupación muy violenta del territorio, la destrucción de ciertos centros ceremoniales y la aparición de nuevas epidemias (sarampión, fiebre amarilla, etc.). Desde los años 1860, algunos franciscanos vuelven a explorar la Selva central y establecen las tres misiones de Quillazú, San Luis y Sogormo entre los yánesha (Smith, comunicación personal, 2018). Pero es realmente a partir de 1920 que se presentó un recrudecimiento de la colonización y la llegada de nuevos agentes religiosos.

La Iglesia Adventista del Séptimo Día llegó al Perú en 1899, institucionalizándose en 1905 gracias al estadounidense Franklin L. Perry. Al año siguiente nació la Misión Peruana y se empezó a reconocer la presencia de algunas agrupaciones adventistas que en esa época eran organizadas a partir del colportaje itinerante 2 (La Serna Salcedo, 2009: 46). En abril de 1919, se iniciaron las actividades educativas con el fin de fortalecer la obra misionera. El Ilamado Colegio Unión permitió la formación de la Universidad Unión Incaica en 1983, la cual desde 1995 se llama Universidad Peruana Unión (La Serna Salcedo, 2009: 47-48). Según La Serna Salcedo, el mayor crecimiento se observó entre 1915 y 1925 (inicio del misionado en la Selva central), entre 1945 y 1955 (expansión de la zona misionera a las zonas andinas y selváticas) y entre 1965 y 1975 (predicaciones masivas y campañas itinerantes en las ciudades). En la zona del Alto Perené, la evangelización estuvo bajo dependencia de la Misión Peruana que se trasladó al Pichis en 1948 como estación misionera central. En 1964, las estaciones y grupos del Pichis pasaron a depender de la Misión Alto Amazonas por problemas de accesibilidad, mientras que las misiones del Perené (zona de Churingaveni, Perené medio) se mantuvieron bajo tutela de la Misión Peruana (subdividida en Misión del Norte, del Sur y del Centro o Misión Andina Central). La Misión del Oriente Peruano es un conglomerado de siete iglesias, entre las cuales encontramos la de Puerto Bermúdez, que permite el acceso al pueblo yánesha (La Serna Salcedo, 2009: 55).

1 En 1631, los franciscanos habían comenzado la evangelización del Alto Huallaga teniendo como centro de operaciones la ciudad de Huánuco y, en 1646, los dominicos entraron a la zona desde la ciudad de Tarma fundando el pueblo de Santo Domingo Soriano en la confluencia de los ríos Palca y Tulumayo (Santos-Granero, 2004: 189).

2 Según La Serna Salcedo, el colportaje itinerante consiste en la venta ambulatoria de textos bíblicos y revistas para financiar las actividades misioneras (La Serna Salcedo, 2009: 45). 
El 24 de mayo de 1922 se aprueba la primera Declaración de Fe y la primera Constitución de la Iglesia en Muquiyauyo (Junín), la cual adopta entonces el nombre de Iglesia Evangélica Peruana (IEP). Esta Iglesia pentecostal forma parte de la Iglesia Universal de Jesucristo (IEP, 2016), que llegó al continente americano gracias a los misioneros británicos a finales del siglo XIX. La primera célula fue fundada en Chucuito (Callao), por doña Federica Petersen, quien compartía el evangelio ya desde 1880. Tratando de resolver los problemas económicos del país, el grupo británico The Peruvian Corporation se comprometió con la construcción y la gestión del Ferrocarril Central3, lo que permitía tener un fácil acceso a la Selva central. Esas vías de comunicación permitieron el auge del proceso de evangelización. Así, el misionero Charles Bright empezó a realizar los cultos en español, iniciando la evangelización en Cerro de Pasco (1914), Jauja (1917) y los pueblos aledaños. En 1919, se constituyó el primer Sínodo Regional de las Iglesias Evangélicas del Perú Central. En 1933, Alfonso Muñoz fundó el Instituto Bíblico Peruano (IBP), hoy llamado Seminario Evangélico de Lima (SEL), y para apoyar la formación de los predicadores en las zonas rurales nacieron los institutos bíblicos (IEP, 2016). En esa época, se produjo la conversión de una parte del pueblo yánesha, lo que debilitó aún más las actividades de los últimos templos. En 1940, la IEP y otras misiones extranjeras constituyeron el Concilio Nacional Evangélico del Perú (CONEP).

En 1946, los misioneros y lingüistas evangélicos del Instituto Lingüístico de Verano (ILV) llegaron al Perú. Fundado como una organización sin ánimo de lucro en 1934 por William Cameron Townsend (1896-1982), pertenece al protestantismo evangélico. En 1956, sus miembros se asentaron en territorio yánesha y aprendieron el idioma (Stoll, 1985: 182). Según Smith, sus trabajos empezaron antes, a fines de los años 1940, específicamente en la zona de Oxapampa, en la comunidad de Tsachopen (Smith, 1981). Desde 1953 formaron a maestros bilingües con el objetivo de evangelizar a la población yánesha. El acuerdo educativo con la república peruana legitimó la construcción de las primeras escuelas bilingües. Con ellas aumentó el número de pastores y de Iglesias. Los yánesha continuaron viviendo bajo la influencia del ILV. La traducción del Nuevo Testamento Yompor po'ñoñ (lit. Biblia) (Duff-Tripp, 1998: 462) a la lengua yánesha fue impreso en 1978 (Liga Bíblica Internacional, 2008). La introducción de una nueva manera de pensar vinculada a la religión cristiana condenaba de una cierta forma la religión yánesha tradicional (Smith, 1981). La representación del alfabeto fue reevaluada en 2011 bajo la autoridad de los mismos lingüistas del ILV (Normalización del alfabeto yánesha RD N 1493-2011-ED)4. En 2015, la Asociación Interétnica de

3 Después de la Independencia del Perú, la gestión del Ferrocarril Central entró en dificultades económicas (bonos de 1865, 1866, 1869, 1870 y 1872). El país estaba casi en bancarrota. En Londres, un grupo formó The Peruvian Corporation para tratar de resolver algunos problemas y recuperar el dinero invertido.

4 En el marco de la implementación de la Ley № 29735 (Ley de Lenguas Indígenas u Originarias), el Ministerio de Cultura registró a dos traductores e intérpretes de la lengua yánesha en 2014 (Base de Datos Oficial de Pueblos Indígenas u Originarios [BDPI]). Incluso, es una lengua considerada en peligro porque no está siendo transmitida a niñas y niños. 
Desarrollo de la Selva Peruana (AIDESEP) «rechazó cualquier intento de imponer una nueva colonización lingüístico-educativa-confesional» y solicitó al Estado peruano no renovar el convenio con el ILV. A pesar de haber sido considerado como una institución que ha violentado a los indígenas con su presión evangelizadora (SERVINDI, 2015), el ILV ha seguido participando en el proceso de evangelización mediante sus trabajos lingüísticos, hasta encontrarse prácticamente inactivo.

En 1977, fecha que coincide con el abandono de los templos yánesha, los dos presbiterios evangélicos yánesha, el de la «parte alta» (Presbiterio Tenomar en la zona de ocupación río arriba o «parte alta», lit. teno) y el de la «parte baja» (Presbiterio Topomar en la zona río abajo o «parte baja», lit. topo), eligieron delegados ante el Sínodo Regional de las Iglesias Evangélicas del Perú Central, el cual congrega a las Iglesias evangélicas indígenas y mestizas de la región (SantosGranero, 2004: 332)5. El CONEP y el SEL están vinculados a la Fraternidad de Asociaciones de Iglesias Evangélicas Nativas de la Amazonía Peruana (FAIENAP), entidad religiosa instalada en Yarinacocha, Ucayali, desde 1988 y registrada en 1995, de la cual forma parte la Iglesia Bíblica Yánesha del Perú (IBYP), institución religiosa desde el 1 de octubre de 2007, ubicada en San Pedro de Pichanaz. La FAIENAP es el organismo representativo de las Iglesias evangélicas de diferentes etnias de la Amazonía peruana. Su creación fue impulsada por el ILV y, junto con la Sociedad Bíblica Peruana (SBP) revisan el Nuevo Testamento y partes del Antiguo Testamento. Entre 2014 y 2016, el consejo directivo de la FAIENAP estuvo a cargo de un presidente yánesha, Augusto Francis Lores, radicado entre San Francisco de Pichanaz y Azulis. El objetivo de la FAIENAP es dar una identidad propia a todas las Iglesias evangélicas nativas. Para logarlo, promueve el intercambio entre las Iglesias de los diferentes grupos étnicos (FAIENAP, 2017a). Por su lado, los presbiterios yánesha se han desarrollado autónomamente6. Según La Serna Salcedo (2009), el envío de misioneros indígenas del Perené al Palcazú existía desde mediados de la década de 1940, con el objetivo de establecer grupos adventistas entre una parte de la población yánesha que venía siendo desplazada hacia esta región como consecuencia de la colonización. En 1950, Vicente Paulino, antiguo maestro y misionero asháninka, accedió a la comunidad yánesha Loma Linda y fundó una misión-escuela que sigue existiendo (La Serna Salcedo, 2009: 68).

Geográficamente, estos movimientos religiosos tuvieron poca interacción. Entre las comunidades yánesha se pueden identificar pueblos evangélicos afiliados a la FAIENAP o a la IEP y pueblos con tendencia adventista7. Las dos doctrinas tienen posiciones teológicas diferentes, lo que genera malentendidos e impide a veces las colaboraciones. Eso produce una cierta escisión entre los pueblos yánesha y, a veces, entre las mismas familias. Estos desacuerdos crean disensiones: cada doctrina se siente atacada por las normas utilizadas por la otra. Por lo tanto, no

5 Existe también el Sínodo del Sur en el centro poblado de Urcos (Cusco).

6 Ciertas Iglesias reformadas son organizadas según un régimen de presbiterio sinodal, basado en el equilibrio entre un consejo local y los delegados nacionales.

7 En algunos grandes pueblos, como Tsachopen, se pueden encontrar Iglesias adventistas y evangélicas. La repartición territorial de esas corrientes religiosas se hace según los sectores comunales. 
pueden, por ejemplo, compartir ciertas celebraciones ni invitarse mutuamente. Principalmente, las causas de los desacuerdos son el consumo de la cerveza de yuca y de ciertos alimentos y la práctica del baile. Asimismo, los exitosos esfuerzos de los misioneros cristianos en esas décadas han tenido efectos devastadores en la práctica de la música (Smith, 1977: 180). A continuación, tomaremos los ejemplos de dos comunidades, una evangélica y otra adventista: la comunidad San Pedro de Pichanaz (sectores Azulis y San Francisco de Pichanaz) y la comunidad Loma Linda (sectores Loma Linda y Puerto Laguna), comunidades que vengo estudiando desde el año 2006.

\section{PRÉDICAS EVANGÉLICAS Y ADVENTISTAS EN LAS COMUNIDADES}

En Azulis, la presencia de la FAIENAP es importante y está relacionada, como vimos anteriormente, con la Iglesia Bíblica Yánesha del Perú (IBYP). Una visita de la IEP realizada en 2015 me permitió constatar que mantienen sus ambiciones. Para dar una identidad propia a las Iglesias Evangélicas Nativas, su objetivo principal es difundir y promover la evangelización con acciones misioneras. «La vida y misión de la Iglesia Evangélica Peruana es predicar el Evangelio (Mr. 16:15), hacer discípulos y bautizar en el nombre del Dios Trino (Mt. 28:19), enseñar y guardar todos los mandatos dados por Cristo (Mt. 28:20), y sanar a todos los que sufren [...] (Lc. 4:8; Mr. 16:17-18; Jn. 20:22-23), de tal manera que magnifiquen el Nombre de Dios [...]» (IEP, s. f.). Para ello, es necesario edificar iglesias nativas donde compartir las experiencias en la extensión del Reino de Dios: «[...] iglesia a la Imagen del Señor Jesucristo y fiel a la Palabra del Dios Trino» (Ro. 8:29; Ef. 4:13; 2Tim. 3:1).

El edificio de culto evangélico de Azulis era viejo, por lo cual se decidió edificar otro (templo parepo', lit. casa del padre; templo Yompor pa'paquëll, lit. casa de Dios8). Una vez terminado, se inauguró con la idea de promover el intercambio y fomentar la confraternidad entre las diferentes Iglesias evangélicas (fig. 2). Se juntaron la Iglesia Alianza Cristiana Misionera del Perú (IACyM) del distrito de Santa Anita (a la cual pertenece la IBYP) y las Iglesias de otras comunidades yánesha, tales como Santa Rosa y Milagro. La iglesia de Santa Anita preparó su viaje a la comunidad Azulis. Allí realizó obras de bien social, obsequió ropa y dictó talleres educativos sobre salud reproductiva y organizó otras actividades sin ánimo de lucro para contribuir a la formación integral humana y cristiana del pueblo nativo y a la superación de la nación, en

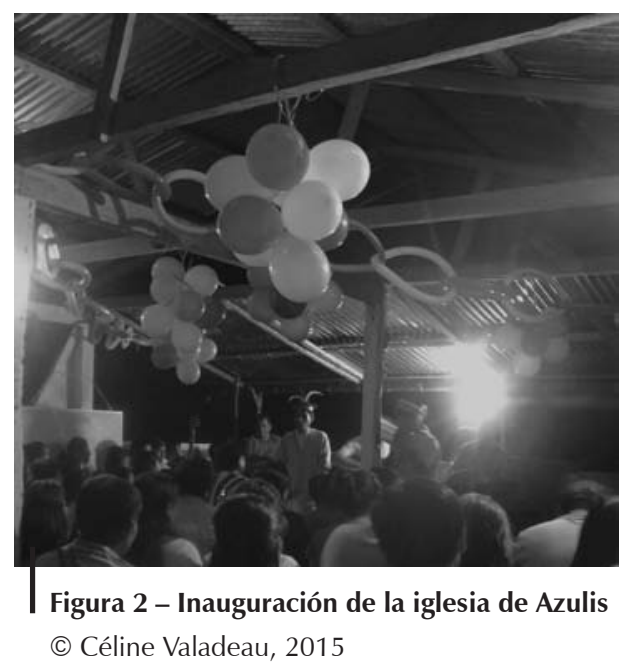

8 «De Nuestro Padre (Yompor)» es la traducción que los misioneros del ILV dan al Dios cristiano. 
su rol de plataforma de animación y de apoyo (FAIENAP, 2017b) (fig. 3). Por otra parte, el grupo de música de la comunidad de Milagro asistió a la inauguración y ayudó, junto con la comunidad de Santa Rosa, en la gestión y aprovisionamiento de alimentos para todos. Durante esta semana, las Iglesias presentes fortalecieron sus relaciones con grupos evangélicos del Perú y de otros países. Es durante estas actividades que se volvió a dinamizar el discurso evangélico, el cual promueve la conversión individual apoyándose en los textos bíblicos. La conversión individual tiene como objetivo permitir el bienestar de la persona. Para lograrlo, son necesarios un cambio de pensamiento fundamental, el rechazo de cualquier tipo de brujería o rituales paganos, el acompañamiento correcto de los muertos y la valorización de una ética conyugal. Además, quien lo logra debe ser implicado en una solidaridad interindividual y difundir el buen pensamiento evangélico. El cambio de comportamiento que uno tiene que realizar le permite tener más iniciativas personales y desarrollar una solidaridad interindividual fuerte.

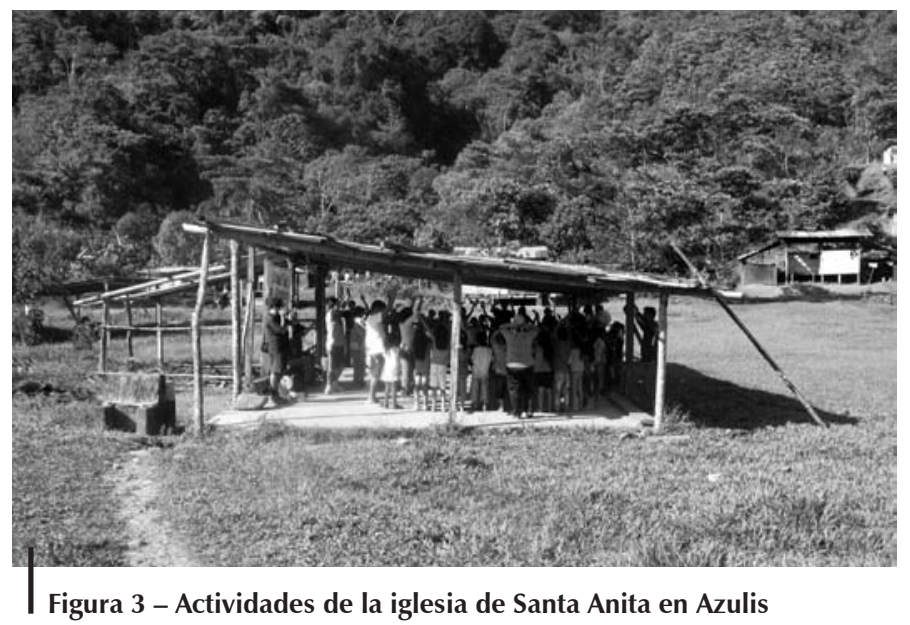

(C) Céline Valadeau, 2015

En los asentamientos adventistas vimos pocas actividades, a diferencia del caso de Azulis. No obstante, los pastores de otras misiones los visitan de manera muy frecuente para predicar. Si no es así, un pastor yánesha elegido por el pueblo asume el rol. En Loma Linda, la misión depende de la Iglesia adventista de Juan Bautista, la cual busca preparar al pueblo para la venida de Jesús, idea que proviene de las profecías específicas de la consolación de Israel (Isaías 40) y del gran día del Señor (Malaquías 4). Muchas de las recomendaciones también están basadas en las escrituras de Elena de White. El modelo profético de esa Iglesia adventista da un énfasis al estilo de vida, especialmente con relación a la comida, la bebida y la vestimenta. La única conducta segura es evitar los lugares de diversión, las bebidas intoxicantes y todo tipo de sexualidad impura. Los cristianos deben vestirse con modestia, decencia, buen gusto, evitando la sensualidad provocativa, «en consonancia con nuestra fe»(De White, 1967: 280). Según ellos, este estilo 
de vida específico es ordenado por Dios y su cumplimiento es primordial para preparar la venida del Señor. Una vida de santificación es necesaria y se debe llegar al punto de reconocer plenamente el poder y la autoridad de la palabra de Dios. Así, se conocerá el crecimiento espiritual, la pureza moral que todo hijo de Dios debe conservar en el corazón (De White, 1892: 68).

Al tomar nota de las diferencias, observamos que la vida cotidiana adventista es mucho más marcada que la de los evangélicos. El día cuenta con actividades definidas que deben ser cumplidas de manera precisa. Existen tiempos dedicados a la religión, a la comida, al trabajo, etc. Las prohibiciones adventistas son también más estrictas que las preconizadas por la Iglesia evangélica. Los adventistas prohíben totalmente las presas de caza ${ }^{9}$, los licores (cerveza de yuca), las hojas de coca y el tabaco. En cambio, la FAIENAP precisa el peligro de tomar un masato masticado por el riesgo de contraer hepatitis $B$, pero no prohíbe la bebida. No obstante, la caza es la actividad de mayor importancia. A pesar de la conversión de la gente al adventismo, esas actividades continúan aún pese a las interdicciones. De la misma manera, los rituales que conciernen a las jóvenes mujeres se siguen haciendo entre las comunidades evangélicas como entre las adventistas (fig. 4). Por otro lado, los evangélicos no se posicionan claramente respecto al asunto de las plantas medicinales; dicen que la Biblia no condena estas prácticas si no son adictivas. La Iglesia adventista admite únicamente los ocho remedios naturales que Dios prescribió para tener una vida saludable y equilibrada, y para la cura de muchas dolencias y sufrimientos (descanso, ejercicio, sol, confianza en Dios, aire puro, nutrición apropiada, sobriedad, agua). La «medicina naturista» es considerada como opuesta al verdadero naturismo y resulta dañina para el cristiano, por desvirtuar y contradecir la palabra de Dios (De White, 1927: 70). Aunque muchos yánesha convertidos al adventismo dicen que ya no usan más las plantas, otros dicen que, en los tiempos míticos, las plantas eran humanos enviados por Dios para ayudar a los yánesha. Entonces, pueden usarlas por ser divinas.

El adventismo estimula más los perfiles individuales o nucleares e impone unas conductas estrictas y hábitos cotidianos precisos, mientras que el evangelismo estimula la cohesión social interindividual. Observamos que, aunque se predican y se recomiendan conductas definidas,

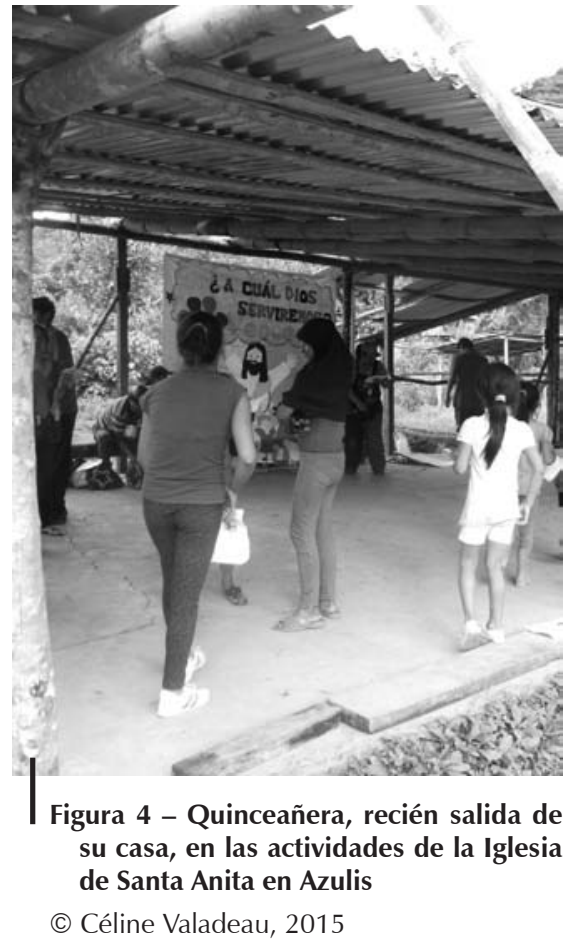

9 Excepto los animales que no rumian y que no tienen el pie hendido y los peces que no tienen escamas, como el pecarí y el mono, entre otros. 
en ciertos casos el pensamiento indígena sobresale: las similitudes entre ciertos elementos que se derivan de la mitología yánesha y los diferentes dichos propuestos por las Iglesias permiten a los yánesha adoptar estos discursos eclesiásticos sin que ello suponga modificaciones particulares en su discurso inicial.

\section{CLIMATOLOGÍA HISTÓRICA EN LA SELVA CENTRAL}

La noción de cuidado ambiental llegó a las comunidades hace décadas con la creación de las zonas protegidas (Parque Nacional Yanachaga-Chemillén en 1986, Bosque de Protección San Matías-San Carlos en 1987, Reserva Comunal Yánesha en 1988 y Reserva de Biósfera Oxapampa-Ashaninka-Yánesha en 2010) y la difusión de la idea de protección del bosque frente a las diversas amenazas. Los programas de manejo forestal y agrícola, como el PEPP (Proyecto Especial PichisPalcazú) o «Bosques en tus manos» del Ministerio del Ambiente del Perú (MINAM, 2010), aportaron también estas ideas de conservación. En general, la noción de protección fue relacionada con tres enfoques principales: contaminación (producción de droga, actividades mineras), necesidad de protección (conservación de las especies silvestres) y manejo sostenible (extracción, agricultura sin quema y de substitución, etc.).

Con la creciente preocupación de cuidar el medioambiente, las Iglesias evangélicas y adventistas tuvieron que posicionarse. Ambas tomaron como referencia el libro de Isaías. Localmente, los evangélicos citan las palabras de Miguel y Pablo Wickham durante las predicaciones, sin aprobar, por lo tanto, las directrices internacionales al respeto. Los autores del libro Ecología y cambio climático: una reflexión cristiana dicen que es necesario desarrollar una conciencia ecológica y que «tenemos que retomar el camino correcto en nuestra sociedad» (Wickham Redman \& Wickham Ferrier, 2012: 80). El argumento dominante, sin embargo, es que Dios dice que tenemos que darnos cuenta de que todo viene de él. Así, evangélicos y adventistas resaltan que «la acción genuina ecológica tiene que salir del centro de nuestra fe. Debe haber un cambio de vida hacia lo sostenible [...]. La crisis ecológica es espiritual, por tanto, necesitamos un arrepentimiento y una conversión a Dios». Subrayan que leer la Biblia permite encontrar respuestas. En 2016, se celebró en Lima el $32^{\circ}$ Aniversario de la Iglesia Evangélica Peruana Santa Anita con estas palabras, posteriormente difundidas en las comunidades yánesha:

Hoy, [...] elevamos nuestro clamor por los estragos del cambio climático en el continente.

Oremos por que cada habitante del continente tomemos conciencia de los efectos adversos del cambio climático producto de no cuidar la naturaleza que Dios creó para que la disfrutáramos y la explotamos irracionalmente. [...]

Oremos por las personas víctimas de las inundaciones producto de la lluvia prolongada, que Dios traiga [...] 
Oremos por que Dios bendiga nuestro continente con estaciones adecuadas y la regularización de nuestro clima y la seguridad de la lluvia en los tiempos necesarios.

Dios de bendición, que creaste los cielos, la tierra y las estaciones del año, te pedimos la bendición [...].

Amén. (23/10/2016)

De la misma manera, Miguel y Pablo Wickham señalan que «en el Pentateuco encontramos más mandatos relacionados con un cuidado adecuado de la naturaleza, como el descanso de la tierra o el reparto adecuado para evitar su explotación especulativa» y que «la Palabra de Dios, que ordena la relación con los demás y con la naturaleza, es una relación en equilibrio». Afirman, finalmente, que este mensaje de restauración de la naturaleza existe en el Nuevo Testamento (Wickham Redman \& Wickham Ferrier, 2012: 112).

Por su parte, los científicos adventistas aceptan las realidades de la microevolución (cambio limitado dentro de una misma especie), pero no las de la macroevolución (cambio de una especie hacia otra diferente). Dios cuida su creación dando a cada especie una capacidad de adaptación genética de acuerdo a la complejidad de su hábitat y a los cambios climáticos. Para estos científicos, la comprensión de la naturaleza aparece coherente con la revelación bíblica. Los adventistas no están a favor del aprendizaje de la creación. Militan por el hecho de que el universo, la tierra, sus recursos y los seres humanos pertenecen a Dios, según lo que dice, por una parte, el Salmo de David y, por otra parte, el Génesis (Génesis 1:27-28). No obstante, Dios confió la gestión de la Tierra a los humanos. En el territorio yánesha podemos observar la posición de la Iglesia Adventista del Séptimo Día con relación a la preservación del medioambiente, del calentamiento global y del cambio climático: «Vemos cómo sucede todo esto y iqué hacemos individualmente para buscar el equilibrio? Somos cristianos y nuestro deber es cuidar de la obra de la creación de Dios. Para nosotros, esa es la principal motivación para cuidar del ambiente en donde vivimos» (Iglesia Adventista del Séptimo Día, 2015). Este punto de vista retomado localmente, nació de una reunión administrativa mundial de la Iglesia adventista en 1995 en los Estados Unidos, en la cual el tema del cambio climático fue objeto de debate, después del acuerdo de Río de Janeiro (Naciones Unidas, 1992). La Iglesia adventista indica también que la profecía de Mateo está tomando cada día más fuerza. Entonces, el pensamiento adventista da importancia a la ecología (Iglesia Adventista del Séptimo Día, 2015).

Allí, las Iglesias evangélica y adventista reconocen el problema climático, con relación a la crisis ambiental, como una consecuencia sin perspectiva de futuro. Los años 2014 y 2015 parecen haber sido cruciales en el desarrollo de las profecías de Daniel y del Apocalipsis. Algunos ejemplos invocados son: el cambio climático en el mundo entero (Mateo 24:7: «Porque se levantará nación contra nación, y reino contra reino; y habrá pestes, y hambres, y terremotos en diferentes lugares») y la violencia por todos lados (Mateo 24:12: «y por haberse multiplicado la maldad, el amor de muchos se enfriará»). Afirman que el capítulo 13 del Apocalipsis es ahora más que nunca nuestra redención y está cerca. La urgencia en torno a las crisis 
ecológicas es una de las profecías bíblicas. Así, nos encontramos con la afirmación de que Dios destruirá «a los que destruyen la tierra» (Apocalipsis 11:18). El cambio climático vino acompañado de otros conceptos como el calentamiento global y los cambios ambientales. Estas nociones fueron también integradas a los discursos evangélicos como factores primordiales de la llegada del fin del mundo por la injerencia de las actividades humanas.

Durante estas últimas décadas, los yánesha escucharon hablar del cambio climático como una razón fundamental para impulsar programas de conservación y manejo del bosque (Larsen, 2015). Apoyada en los discursos religiosos, la noción de cambio climático tuvo también resonancia con sus observaciones sobre el medioambiente y en el clima, dando así la evidencia de un problema global y de mayor gravedad.

\section{EL CAMBIO CLIMÁTICO COMO PROCESO DE TRANSFORMACIÓN INTEGRAL}

Ahora bien, ¿cómo los yánesha hablan del cambio climático?

Analizar las estaciones del año nos permite definir los regímenes climáticos locales de cada una, es decir, especificar las características de una temporada. El carácter de cada una de las estaciones es relativamente estable y se nota que puede ser modificable local y temporalmente por acciones antrópicas (generar el aumento de la temperatura en un terreno por talar árboles o desaguar pantanales para sembrar). Para los yánesha, el clima puede cambiar con estos esquemas sin mayor problema. De manera general, el ciclo anual está determinado por dos estaciones, seca (charo) y de lluvia (huapo). Los nombres de las temporadas del calendario yánesha «son los de las lunas llenas que se determinan según la posición de ciertas constelaciones de estrellas cuando aparecen las lunas llenas» (R. C. Smith, comunicación personal, 2018). Numerosos indicadores permiten predecir estas épocas, lo que permite a los yánesha organizar sus actividades productivas y definir el calendario agrícola y las temporadas de caza y de pesca. Pero desde hace algunos años, ciertas aves — como las golondrinas (maths) — se equivocan. Normalmente anuncian la época seca (chechemh, lit. marzo; coquemh, lit. abril) y se van junto con otras golondrinas _llamadas shellenen-, indicando así la llegada de la temporada lluviosa (o'cayonquëmh, lit. setiembre; huorocteshemh, lit. octubre). A partir de la época yellemh, que corresponde a los meses de abril y mayo — y hasta el mes de diciembre (huancahuallaquëmh)_, es posible cazar de noche. Pero ahora, durante esos meses, se nota que algunos animales no tienen el peso que deberían tener. Por ejemplo, los agutíes (Cuniculus y Dasyprocta) no se encuentran «gordos» en el mes de setiembre, ni los armadillos (Dasypus) o los coatís (Nasua) en agosto (huarcaphsemh). Además, ciertos animales engañan a los cazadores con gritos raros dando la impresión de que ciertas presas no son animales reales, sino encarnaciones de espíritus. Eso sucede usualmente cuando «los Mellañotheñ [...] no nos reconocen aunque estamos pintados de achiote (Bixa Orellana L.)» o cuando los animales están siendo cazados de manera abusiva: 
«lo que sucede es que a veces no se puede cazar nada, unos dicen que los Mellañotheñ no logran guardar los animales en sus cerros porque se volvieron como locos», explica un señor yánesha. En lo que concierne a los peces, no aparecen tan «gordos» como antes, especialmente como sí lo eran en la época llamada chechemh, y los huevos que algunos ponen en noviembre son ahora muy pocos. Hoy, este ciclo anual no corresponde al comportamiento que las especies naturales deberían tener normalmente. Los yánesha hablan de equivocaciones en el comportamiento de ciertas especies.

En cuanto a la producción agrícola, la estrella Antares, chemuellem, posicionada al cenit al amanecer a finales del mes de marzo, y Las Pléyades, Oncoy, posicionadas al cenit al alba, siguen indicando el final del verano. La posición de la primera, por ejemplo, permite iniciar la siembra de coca (Erythroxylum spp.), frejoles (Phaseolus sp.) y cacahuetes (Arachis sp.). «Pero, ¿cómo sembrar cuando las épocas de verano y de lluvia no son conformes?» (tantos días de calor como de lluvia: «Errponmatcha' charerrerr»), «parece que los insectos se equivocan». Se dice que los chapulines (Acrididae) y las ranas (Ranidae) no logran cantar para avisar la llegada de la lluvia; a veces cantan en cualquier momento — cuenta el encargado de la Iglesia del Séptimo Día de Loma Linda-. Así, las plantas no logran crecer como deberían, se pudren y a veces, debido a una creciente no prevista, el río se las lleva. Las plantas de las chacras no son las únicas en estar «confundidas». Por ejemplo, algunas plantas indicadoras al borde del camino no reaccionan de manera adecuada. La mimosa púdica no paraliza a los enemigos, tampoco las juncias (Cyperaceae) avisan de la dirección de las presas de caza. El vegetalista encargado de la Iglesia evangélica de Azulis constata también que unas plantas utilizadas para mejorar las capacidades personales ya no hacen efecto. Unos niños que fueron tratados con plantas para ser ágiles tardaron mucho en caminar y saltar. Además, muchos recién nacidos no logran vivir y nacen en enero (yoncayanquëmh), considerada generalmente una mala época para dar a luz — explica otro vegetalista afiliado a la Iglesia del Séptimo Día de Tsachopen-.

Muchos cuentan que aumenta el peligro de caminar en el bosque: los Mellañotheñ se ponen bravos y los espíritus malos que habían alejado vuelven a acercarse. Así, unos no vuelven o se encuentran río abajo. En 2011, la esposa del encargado de la Iglesia evangélica de Azulis contó cómo el jefe de la comunidad de Santo Domingo fue ahogado por los espíritus del río: «se pusieron bravos por el nivel del agua» (que no corresponde al de la estación seca). La coca, que permite adivinar sobre un viaje gracias a la combinación de la disposición de las hojas sopladas y de su sabor, no ayuda ahora sino en pocos casos. Asimismo, las otras adivinaciones con hoja de coca se vuelven difíciles y algunos ya no quieren practicarlas por temor a equivocarse. De la misma manera, el tabaco ya no contribuye a proteger las casas de los espíritus que vuelven, ni permite las curaciones en ciertos casos. Unos dicen que «es como si se hubiera vuelto tabaco falso, pero que sí hay personas que todavía tienen unas matas reales».

La inoperancia e inadecuación de los comportamientos de los vegetales, animales y espíritus fueron comparadas con la presencia de un insecto palo, cherak (Phasmida), 


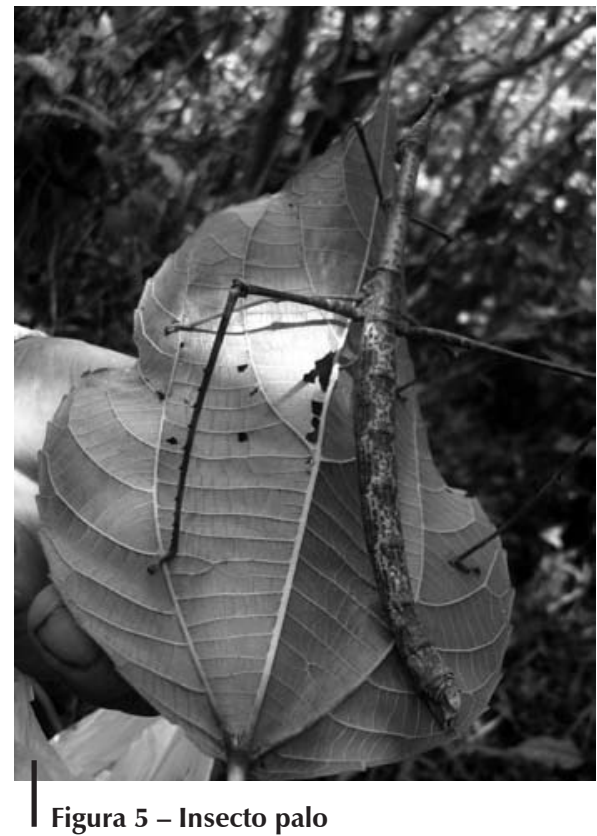

(C) C. Valadeau, 2007

encontrado un día de caminata: «Mira este, este gusano, el que está dentro, no alcanza a parecer a lo que debería. Estos normalmente no se deben ver, y aquí tan cerca, peor» (fig. 5). Estas equivocaciones, además de los indicadores que no coinciden con los movimientos de las estrellas, los cuales son cruciales para los yánesha en su organización productiva, permiten afirmar que hay un cambio real. Como indicó SantosGranero, a pesar de la evangelización masiva, estos indicadores siguen teniendo una gran importancia (Santos-Granero, 2004: 250). Según muchos, todos estos elementos son prueba del cambio climático que se define como una serie de frustraciones y de disconformidades en lo que normalmente se sabe del entorno y de las distintas especies que viven allí. Existe una mala comunicación entre las especies del mundo, lo que implica que las relaciones entre ellas parezcan desordenadas. Un día una señora me dijo: «Parece que entramos en un nuevo conflicto. El sufrimiento de la gente aumenta por castigo, la brujería también, hay muchos problemas y muchos muertos» 10 . Asimismo, dijo que no se debe llegar a lo mismo que ocurrió en la época del gran diluvio (apelleche') y de la oscuridad (a'checmetamhphsarethteñets, lit. haber sido puesto en la oscuridad). Este momento se caracterizó por la ausencia de la reproducción biológica, pues la falta de recursos vegetales dificultó el dar a luz y obstaculizó la fabricación de la persona yánesha por no tener los componentes vegetales y animales adecuados, lo que imposibilitaba la construcción de los humanos (Valadeau, 2012). El período actual es semejante al transcurrido en los tiempos antiguos cuando:

Yompor Rreth era una divinidad mala, sin compasión, ni generosidad, ni amor. A él le gustaba hacer sufrir al pueblo yánesha. A veces, no alumbraba la tierra, imponía así una eternidad sin luz. Otras veces, botaba piedras desde el cielo y producía catástrofes y temblores, tormentas e inundaciones. Esta época de caos, de muerte y de destrucciones era una época muy peligrosa. Hubo muchos conflictos, asesinatos, brujería y los niños no lograban nacer (Valadeau, 2012: 299).

Estos elementos de la mitología explicitan que empezó una nueva época después del período mitológico (mellapoterrmocha). Esa nueva época corresponde al

10 Los casos de brujería están aumentando: en setiembre de 2016 en el Alto Shiringamazú, Rosa Villar Jarionca, de 73 años, fue quemada viva por haber provocado dolores de barriga y cólicos (The Guardian, 2016). 
fin de los tiempos (mellapoteñets). En los salmos y prédicas, muchos evocan los problemas climáticos. Cuando los yánesha $-y$, entre ellos, representantes de distintas Iglesias - relacionan estos problemas ambientales con el desorden creciente de las comunicaciones entre las especies vivas que observan, dicen que este caos puede señalar el principio de un desastre grande. Algunos piensan en un tipo de mensaje escatológico. Las relaciones entre los seres vivos no parecen articularse de una manera normal. Por lo tanto, parece que el ser humano está en peligro: no puede garantizar que los procesos de construcción ritual de la persona humana sean realmente efectivos. Por ello, el cambio climático fue entendido no como una variación usual, sino como un cambio generalizado: una forma de transformación integral del mundo que puede producir caos si la comunicación entre especies y divinidades no logra ser eficiente.

\section{CHAMANIZACIÓN DE LAS NUEVAS PRÁCTICAS RELIGIOSAS}

La prédica evangelizadora del ILV tuvo un impacto cierto entre la gente yánesha, pero al mismo tiempo confuso por haberse apropiado de su propia terminología. Por ejemplo, al tratar de dar sentido a las enseñanzas evangélicas en términos de sus propias categorías, los lingüistas designaron al «Dios Sol» (Yompor Ror) para nombrar a la trinidad cristiana, sembrando así confusión entre la gente (Smith, 1981). De la misma manera, el término Mellañotheñ fue utilizado para hablar de los ángeles («el ángel viene de Dios» traducido por mellañotheñ huena Yomporeshoth) (Duff-Tripp, 1998: 229), aunque los yánesha los identifican como espíritus tutelares. El mensaje religioso evangélico y adventista fue construido con importantes paralelos con el discurso religioso yánesha. El Nuevo Testamento en lengua yánesha, traducido por el ILV, incluye una parte del vocabulario religioso tradicional (Smith, 1981). «Adoptaron el término coshameteñets (lit. alabar a las divinidades y expresar alegría a través de la ejecución de la música sagrada coshamhñats) para hacer referencia al acto de alabar y rendir culto al dios cristiano en la iglesia, mediante la entonación de himnos religiosos» (Santos-Granero, 2004: 321). Además, algunos de «los encuentros religiosos organizados por las congregaciones evangélicas [...] son convocados de modo que coincidan con noches de luna llena. Al igual también en el caso de las ceremonias organizadas por los antiguos cornesha' [...] se invitaba a fieles, predicadores y pastores de otras comunidades» durante varios días al igual que la antigua organización, en la cual los antiguos kornesha' elevan plegarias y se dirigen a los fieles mediante sermones. Los presentes oran y entonan himnos religiosos (Santos-Granero, 2004: 334). Aquí se ve que las transmutaciones de conceptos religiosos han sido posibles en la época del ILV y que siguen siéndolo. Hoy, hace falta la comunicación con la divinidad Yompor Ror y la situación de desorden generalizado debido al «cambio climático» vuelve a ser desfavorable, incluso se convierte en un riesgo vital. Para los yánesha, frente a este vacío, ciertos elementos de los discursos religiosos evangélicos y adventistas pueden relacionarse directamente con la posibilidad de establecer una comunicación con Dios (yánesha), procesando así otro tipo de transmutación. 
Por otro lado, en la actualidad, las personas encargadas del funcionamiento de las Iglesias en las comunidades son los mismos conocedores del cuidado de la persona humana, en numerosos casos son los vegetalistas, en otros casos son los chamanes, o ambos. Aquí la distinción entre las categorías chamán y vegetalista está desapareciendo. Uno de ellos dice que la comunicación no se ha vuelto a establecer en las últimas décadas por haberse equivocado de interlocutor divino (entre el Dios cristiano y el Dios Sol) y por no tener más a los antiguos kornesha'. Después de más de tres décadas, hubo algunos chamanes/vegetalistas encargados de Iglesias que se autoproclamaron kornesha', retomando así la definición antigua de la palabra y juntando a más gente para fortalecer sus «nuevos templos» (lit. parepo': iglesia o templo). Estos nuevos «antiguos kornesha'» autoproclamados pasan de comunidad en comunidad para ampliar su influencia. En Tsachopen, un señor me dijo que son kornanesha" "los descendientes de los kornesha' del pasado». Al verlos llegar a sus comunidades, la gente evalúa si conocen los sitios exactos de los antiguos templos, como prueba de sabiduría. Se presentan como sabedores que han estado en aislamiento y que conocen del consumo de coca y de tabaco y de las canciones sagradas. Gracias a sus capacidades, pronto podrán hallar a las divinidades y dioses que se callaron. Ellos representan, entonces, la posibilidad de poder volver a asegurar una comunicación correcta entre las especies vivas. La mayoría de los que conozco son adventistas. No obstante, en esta coyuntura, la escisión que ha podido aparecer entre evangélicos y adventistas tiene tendencia a borrarse.

La urgencia de la situación aparece como una fuerza unificadora y, como en la antigüedad, los miembros de una misma parcialidad pueden constituirse en seguidores de diferentes antiguos kornesha' o, actualmente, de diferentes autoridades religiosas (Santos-Granero, 2004: 262). Anteriormente, el área de influencia de los antiguos kornesha' no era circunscrita territorialmente y la unión de sus miembros se hacía en adhesión a los mensajes divinos sin que unos lazos de parentesco o de vecindad se tuvieran en cuenta (Santos-Granero, 2004: 265). Hoy, en momentos de crisis, la voluntad de recuperar el contacto con Yompor Ror parece ser de la mayor importancia. A consecuencia de un tipo de profetismo, y por el impulso de algunos nuevos antiguos kornesha' encargados de Iglesias, unos grupos constituidos de evangélicos y de adventistas migran de los distintos pueblos (como Tsachopen) hacia las tierras más bajas del Atalaya para establecer una nueva comunidad y volver a encontrar la red de comunicación adecuada que debe normalmente existir entre los seres vivos.

Asimismo, el desorden a nivel de las federaciones les hace perder su autoridad política por malentendidos internos y divisiones. Entonces, las autoridades políticas internas de las comunidades, sus juntas directivas, optan por escuchar a las nuevas autoridades religiosas. Por lo tanto, y de manera coherente, las autoridades religiosas yánesha son solicitadas y detentan una forma de influencia, que junta poderes religiosos y políticos, como en el pasado.

Si quedó claro que la definición de la palabra [antiguo] kornesha' cambió hasta perder su dimensión religiosa con los kornesha' contemporáneos, hoy parece que 
kornesha' tiene un doble sentido: uno emparentado con el poder político de los líderes (kornesha' contemporáneo) y otro emparentado con una autoridad religiosa y política (nuevos antiguos kornesha'). Podríamos ir en este sentido, considerando las ideas de Capredon — quien concluyó, sobre el cristianismo en un grupo de indígenas brasileños, que los movimientos evangélicos parecen haber sido dejados como tal para volverse un conjunto de prácticas propias (Capredon, 2016: 627)—. Entre los yánesha, parece que la figura del antiguo kornesha' volvió a aparecer a través de unos chamanes o vegetalistas encargados de Iglesias, los que hemos llamado los nuevos antiguos kornesha' y que estas figuras en realidad cumulan el cargo religioso y político, como los antiguos kornesha', más la capacidad de sanar o hechizar de los chamanes o vegetalistas. En este proceso, y como una hipótesis que se podría profundizar, la chamanización de las nuevas prácticas religiosas consistiría en el alejamiento progresivo de las ideas evangélicas y adventistas para volver a representaciones propias. De pronto, está naciendo una nueva figura en la vida político-religiosa yánesha.

\section{Referencias citadas}

AMICH O. F. M., J., 1975 - Historia de las misiones del convento de Santa Rosa de Ocopa, 554 pp.; Lima: Milla Battres.

BENAVIDES, M. \& PARIONA, M., 2002 - La Cooperativa Forestal Yanesha y el sistema de manejo forestal comunitario en la selva central peruana. In: El cuidado de los bienes comunes: gobierno y manejo de los lagos y bosques en la Amazonía (R. C. Smith \& D. Pinedo, eds.): 353-384; Lima: Instituto de Estudios Peruanos (IEP), Instituto del Bien Común.

CAPREDON, E., 2016 - Les Églises autonomes. Évangélisme, chamanisme et mouvement indigène chez les Baniwa de l'Amazonie brésilienne, 672 pp.; París: École des Hautes Études en Sciences Sociales (EHESS), Universidade do Estado do Rio de Janeiro. Tesis de doctorado en Antropología social y Etnología.

DE WHITE, E. G., 1892 - El camino a Cristo. Conozca los pasos a seguir para alcanzar la paz interior, la seguridad de la salvación y una plena transformación en Cristo, 128 pp.; Doral: Asociación Publicadora Interamericana.

DE WHITE, E. G., 1927 - Joyas de los Testimonios, Tomo 2. Consejos para la iglesia seleccionados de los testimonios, 287 pp. Disponible en https://adventistasrd. interamerica.org/uploaded_assets/162969

DE WHITE, E. G., 1967 - Mensajes selectos, Tomo 3. Disponible en https://text.egwwritings. org/publicationtoc.php?bookCode $=3 \mathrm{MS}$

DUFF-TRIPP, M., 1998 - Diccionario Yanesha' (Amuesha)-Castellano, 688 pp.; Lima: Ministerio de Educación, Instituto Lingüístico de Verano (ILV).

FRATERNIDAD DE ASOCIACIONES DE IGLESIAS EVANGÉLICAS NATIVAS DE LA AMAZONÍA PERUANA (FAIENAP), 2017a - Visión y misión de la FAIENAP. Disponible en https://faienap.wordpress.com 
FRATERNIDAD DE ASOCIACIONES DE IGLESIAS EVANGÉLICAS NATIVAS DE LA AMAZONÍA PERUANA (FAIENAP), 2017b - Propósito y fines. Disponible en https:// faienap.wordpress.com/faienap/proposito-y-fines/

IGLESIA ADVENTISTA DEL SÉPTIMO DÍA, 2015 - Hablando de Esperanza: Los adventistas y el medio ambiente. Disponible en https://videos.adventistas.org/es/editoria/biblia/ tema-3-los-adventistas-y-el-medio-ambiente-hablando-de-esperanza/

IGLESIA EVANGÉLICA PERUANA (IEP), 2016 - Nuestra historia - Breve historia de la iglesia evangélica peruana. Disponible en https://fr.scribd.com/document/311720951/ Brebe-Historia-de-La-Iglesia-Evangelica-Peruana

IGLESIA EVANGÉLICA PERUANA (IEP), s. f. - Misión y visión de la lglesia. Disponible en http://www.iglesiaevangelicaperuana.org.pe/site/nosotros/

LA SERNA SALCEDO, J. C., 2009 - Más allá de la parusía: el enfrentamiento al demonio en el bosque: religión, política y sociedad asháninka a través de la presencia misionera adventista en la selva central peruana (1920-1990), 213 pp.; Lima: Universidad Nacional Mayor de San Marcos (UNMSM). Tesis de Magíster en Ciencias de la Religión.

LARSEN, B. P., 2015 - Post-Frontier Resource Governance: Indigenous Rights, Extraction and Conservation in the Peruvian Amazon, xiv + 185 pp.; Londres: Palgrave Macmillan.

LIGA BÍBLICA INTERNACIONAL, 2008 - Yompor po'ñoñ ñeñ̃ attõ yepartseshar Jesucristo e'ñe etserra a'poctaterrnay Yomporesho / El Nuevo Testamento de nuestro Señor Jesucristo en el idioma yanesha', 682 pp.; Buenos Aires: Wycliffe, Liga Bíblica Internacional. Disponible en https://www.scriptureearth.org/data/ame/PDF/00WNTame-web.pdf

MINISTERIO DEL AMBIENTE DEL PERÚ (MINAM), 2010 - Resolución Ministerial N..$^{\circ}$ 126-2010-MINAM; Lima. Disponible en http://www.minam.gob.pe/wpcontent/uploads/2013/09/rm_126-2010-minam.pdf

NACIONES UNIDAS, 1992 - Convención marco de las Naciones Unidas sobre el cambio climático, 26 pp.; Río de Janeiro. Disponible en https://unfccc.int/resource/docs/ convkp/convsp.pdf

QUINTANA, M., 2004 - Les chemins de la foi - les chemins du rachat. L'œuvre évangélisatrice des Mercédaires au Pérou du XVle au XVIle siècle. Caravelle. Cahiers du monde hispanique et luso-brésilien, 82: 41-61.

SANTOS, F., 1986 - Bohórquez y la conquista espúrea del Cerro de la Sal: Tres versiones y una historia. Amazonía Peruana, VII (13): 119-134.

SANTOS-GRANERO, F., 1998 - Writing History into the Landscape: Space, Myth, and Ritual in Contemporary Amazonia. American Ethnologist, 25 (2): 128-148.

SANTOS-GRANERO, F., 2004 - Los Yánesha. In: Guía Etnográfica de la Alta Amazonía, Volumen IV: Matsigenka, Yánesha (F. Santos \& F. Barclay, eds.): 159-359; Balboa, Lima: Smithsonian Tropical Research Institute, Instituto Francés de Estudios Andinos (IFEA).

SERVINDI, 2015 - Perú: Rechazan colonización «lingüístico-educativa-confesional» del ILV. Disponible en https://www.servindi.org/actualidad/137140

SMITH, R. C., 1977 - Deliverance from chaos for a song: a social and religious interpretation of the ritual performance of Amuesha music, xi + 326 pp.; Cornell: Graduate School of Cornell University. Tesis de doctorado.

SMITH, R. C., 1981 - The Summer Institute of Linguistics: Ethnocide Disguised as a Blessing. In: Is God an American? An Anthropological Perspective on the Missionary Work of the Summer Institute of Linguistics (S. Hvalkof \& P. Aaby, eds.): 121-132; 
Cambio ambiental y chamanización de las nuevas prácticas religiosas entre los yánesha

Copenhague: International Work Group for Indigenous Affairs (IWGIA), Survival International.

SMITH, R. C., 1999 - Caciques chinchaycochas, funcionarios incas y sacerdotes amueshas: los caminos antiguos de Chinchaycocha hacia la selva central. Cultura Andina, 3: 59-71.

STOLL, D., 1985 - ¿Pescadores de hombres o fundadores de Imperio?, 496 pp.; Lima: Centro de Estudios y Promoción de Desarrollo (DESCO).

THE GUARDIAN, 2016 - Woman burned as a witch in Peruvian rainforest, prosecutor says (28 de septiembre de 2016). Disponible en https://www.theguardian.com/ world/2016/sep/28/woman-burned-witch-peru-rainforest-prosecutor

VALADEAU, C., 2012 - Médecine chez les Yanesha d'Amazonie péruvienne. La traversée par les plantes, 386 pp.; París: L'Harmattan.

WICKHAM REDMAN, M. J. \& WICKHAM FERRIER, T. P., 2012 - Ecología y cambio climático. Una reflexión cristiana, 189 pp.; Barcelona: Andamio. 